\title{
A HYBRID VEHICLE CONFIGURATION WITH ZERO EMISSION
}

\author{
José Manuel Andújar Márquez \\ andujar@diesia.uhu.es
}

Manuel Jesús Vasallo Vázquez

Francisca Segura Manzano

francisca.segura@diesia.uhu.es

manuel.vasallo@diesia.uhu.es

\begin{abstract}
Dpto. Ingeniería Electrónica, Sistemas Informáticos y Automática, Universidad de Huelva, E.P.S. La
Rábida, Carretera Huelva-La Rábida s/n, 21071 Palos de la Frontera (Huelva)
\end{abstract}

\begin{abstract}
The search for alternatives to petroleum economy, in automotive field, must satisfy the energy requirements and to offer, at least, the same performance than conventional vehicles. This work proposes a vehicle configuration that satisfies its real energy requirements and also obtains a zero level of noxious emissions. This topology is constituted by a fuel cell, a battery and a bank of ultracapacitors. Once the energetic supply is guaranteed, the second problem consists on the power delivery control strategy. The importance of this control is to optimize the hybrid vehicle components operation mode. By means of simulations, this work shows the deficiencies of current control systems with respect to the supply and the energetic efficiency. Moreover, it suggests using a fuzzy control in the proposed topology in order to optimize the efficiency. This control policy is currently being developed by the author.
\end{abstract}

Key words: electric vehicle, fuel cell, fuzzy control.

\section{Introduction}

The traditional fuels, as the derivatives of the oil, present a larger number of disadvantages than advantages. Besides the political, social and economic conflicts that have happened in the last decades, it is necessary to add real facts as the high level of emission of carbon dioxide and carbon monoxide and the direct consequences on the planet as the thaw of the glacial ones, the floods, the acid rains and the greenhouse effect.

The Transport Sector produces $22 \%$ of the emission of $\mathrm{CO}_{2}$ calculated between 1300 and 7700 million tons per year. From the point of view of the safety, the petrol and combustible diesel oil, that even being more used, are also the most dangerous in case of accident.

In order to improve the fuel consumption, to reduce the noxious emissions, as well as to offer a more economic vehicle without sacrificing neither the security nor the performance, hybrid technology is being object of study and development in the framework of automotive industry.
"Hybrid" concept is often used for those configurations of vehicles which result from a combination between an internal combustion engine and an electric motor, and also for those vehicles that, using only an electric motor, have another element that provides additional energy to the battery.

There are two different basic topologies in electrical hybrid vehicles.

\section{A. Series hybrid}

The total energy on board results from the combination of two or more power sources (figure 1). The internal combustion engine (ICE) connects to a generator (GEN) to produce electrical power. This electric power is stored in a battery (BAT). The electrical motor (EM) works with $\mathrm{AC}$ current, so an inverter (INV) is necessary to turn the $\mathrm{DC}$ current to AC current. The electric motor supplies the traction power to wheels across a mechanical differential gear. Therefore, the traction is obtained from an only central electric motor.

The continuous arrows indicate the energy flow, and the discontinuous arrow for regenerative braking flow. The regenerative braking is a method to take advantage of the vehicle's kinetic energy during the braking.

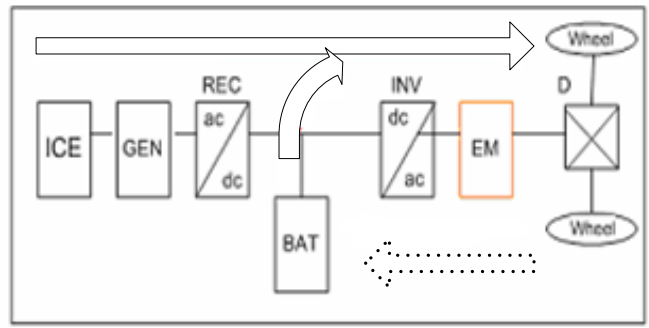

figure 1. Series Hybrid topology

\section{B. Parallel hybrid}

This topology is a combination of traction systems: an electric motor and an internal combustion engine work jointly (figure 2). The power distribution is just mechanical. The internal combustion engine connects directly to a gear box (GB) to produce mechanical power. The energy stored by the battery converts to $\mathrm{AC}$ by a inverter. Then the electric motor transforms the electric power to mechanical power. There are three working ways: 1- the gear box can receive power from two sources (internal combustion engine and battery), 2- someone of 
the sources is off, 3- the internal combustion engine drives only the electric machine to charge the battery.

The continuous arrows indicate the energy flow, and the discontinuous arrow for regenerative braking flow.

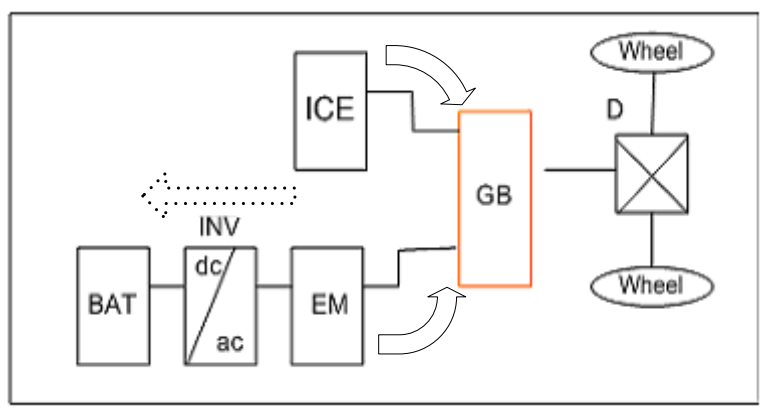

figure 2. Parallel Hybrid topology

It is visible that in all these topologies the energy path for regeneration comprises the electrical traction motor, the traction inverter and the battery.

Any topology different to these previously mentioned ones, is called "complex hybrid topology". Hence, series hybrid configurations with power peak units, parallel hybrid configurations with flywheel or combined hybrid vehicles, are included in this complex hybrid topology. The topology of a combined hybrid vehicle is a combination of a series and a parallel hybrid topology. The connection between the series hybrid part and the parallel hybrid part is purely mechanical. This solution takes advantage of the two basic topologies, series and parallel.

This work proposes a series hybrid configuration that is composed of a fuel cell, a battery and a bank of ultracapacitors and that supplies an electric motor. This configuration guarantees the energetic supply. The system is being installed in the laboratory without integrating the components as a small car. The objective is the study of each component and the synthesis of a suitable control system.

Besides the power sources in the hybrid topology, a suitable power distribution system is also fundamental. The control strategy plays a basic role because it has influence on the global energetic efficiency and on the fuel consumption. Currently, such as our simulations have also corroborated, the control strategies developed in series hybrid topologies with fuel cell do not satisfy simultaneously the power demand and the optimum energetic efficiency of the electrical components. Moreover and in order to solve these deficiencies, the authors are developing fuzzy control policies based on machine learning of models by neuro-fuzzy techniques and on non-lineal control strategies. The current fuzzy control applications are related to configurations that combine an internal combustion engine with an electric motor [3]. Known the advantages of fuzzy logic in multiple problems, it can be expected interesting results in the proposed topology.

Section 2 describes the power sources in the topology. In section 3, performances of the two following topologies are compared by means of simulations: one, the topology composed of a fuel cell and a battery, and the other one, the topology consisted on a fuel cell, a battery and a bank of ultracapacitors. The proposed topology scheme is presented in section 4 and finally, in section 5 , the main conclusions and the current research lines are indicated.

\section{Description of components}

The system proposed in this work belongs to a series hybrid topology. The energy sources are a fuel cell, a battery and a bank of ultracapacitors.

\section{A. Fuel Cell}

It is the basic element of energetic supply of the vehicle. The classification of the different fuel cells is done on the basis of a series of criteria. The electrolyte that is used in the chemical reaction, the used fuel, the temperature of operation and the maximum efficiency are the aspects that determine the election of the fuel cell that is going to be used.

-The polymer electrolyte fuel cell (PEMFC) has a temperature of low operation to $100^{\circ} \mathrm{C}$ and an efficiency of $60 \%$.

-The alkaline fuel cell (AFC) uses as electrolyte $\mathrm{KOH}$, its temperature of operation is about $120^{\circ} \mathrm{C}$ and its efficiency is around $60 \%$.

-The phosphoric acid fuel cell (PAFC) works about $200^{\circ} \mathrm{C}$ with an efficiency of between $50-80 \%$.

-The carbonates bankrupts fuel cell (MCFC) uses lithium carbonate(Li2CO3) and potassium carbonate (K2CO3) as electrolyte, the temperature of operation reaches about $650^{\circ} \mathrm{C}$ with an efficiency of among 60-90 $\%$.

-The oxides solid fuel cell (SOFC) uses $\mathrm{Zr}$ as electrolyte, the temperature of operation raises even more than $1000^{\circ} \mathrm{C}$ with an efficiency of between $60-90 \%$.

The proton exchange membrane fuel cell (PEMFC) emerges as one of the most promising candidates, both for stationary and automotive applications, to replace the traditional systems of internal combustion. The PEM fuel cell offers a high electrical efficiency (60-70\%) in comparison with an internal combustion engine (20-30\%). It's especially attractive for automotives applications, because of its high power density ( power per active area of fuel cell ) and its low operating temperature in comparison with other fuel cells.

The main restriction in the fuel cell operation can be observed from the curve of maximum efficiency. Figure 3 shows that there is a power range where the PEM fuel cell 
works around the highest value of efficiency. Out of this range, it is possible to extract power from the fuel cell, but with a smaller efficiency. Therefore, it is not possible to demand any value of power because it is not interesting to work outside this range from a practical point of view. As a consequence, it is essential to incorporate another energy source.

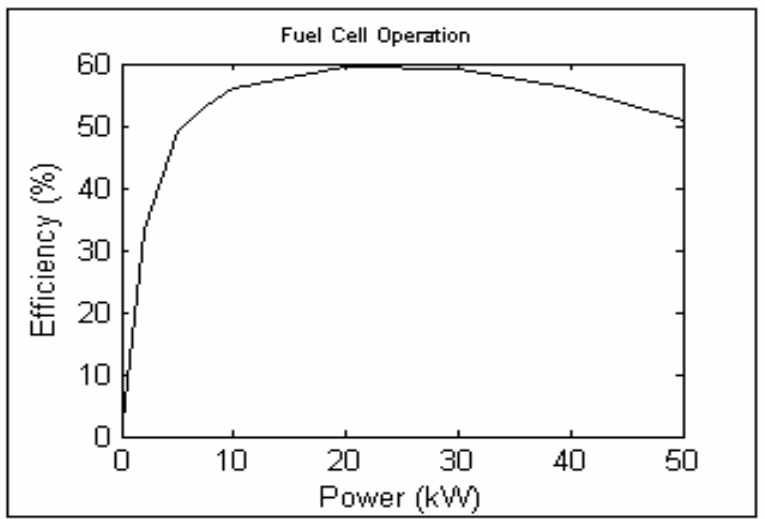

figure 3. Fuel Cell Efficiency curve

\section{B. Battery}

The purpose of using the battery is to reduce the difference between the power supplied by the fuel cell and the power required by the engine, which achieves high values in phases of acceleration.

There are batteries composed of lead - acid (PbSO4), of nickel - cadmium (Ni- Cd), of nickel - hydride (Ni-H), of bromine - zinc (Br-Zn), of sulphur - sodium (NaSO4), of sodium - chloride $(\mathrm{Na}-\mathrm{Cl})$, of nickel $(\mathrm{Ni})$ or of lithium (Li). The parameters to take into account are, between others, the energetic density, the specific power, the temperature of operation, the average life time and the cost of maintenance. Attending to these aspects and to the availability of the market, the batteries of lithium and of nickel - cadmium reach the requirements of an electrical vehicle referring to the energetic density and power density.

It is not possible to demand high power levels in short time periods because it supposes an increase of the losses and the operating temperature, as well as a decrease of the estimated average life time. Figure 4 shows the variation of the power supplied by the lithium battery versus the charge state. It can be observed how this power is limited.

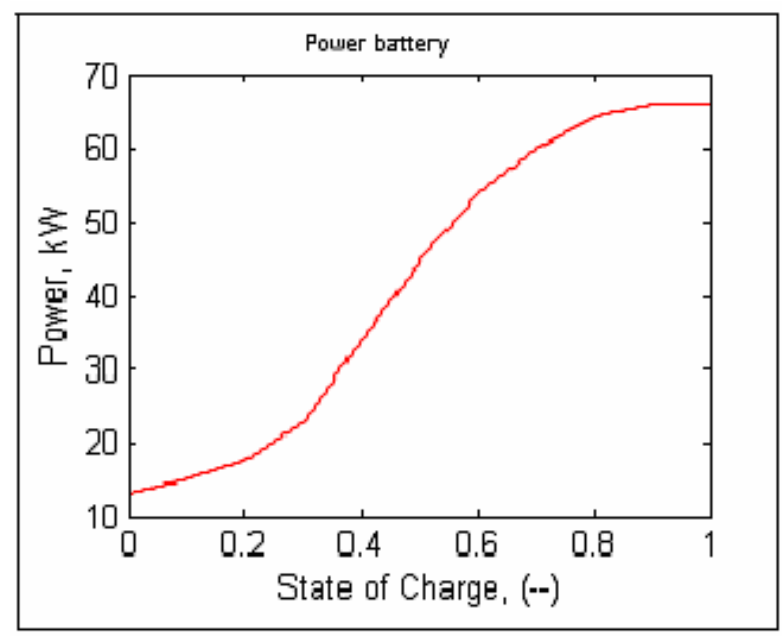

figure 4. Maximum Battery Power

\section{Ultracapacitor}

This device covers, in short time periods, the peaks of maximum power demand. Therefore, it is essential to include this device; the fuel cell together with the battery can not provide all the power required by the other engine.

The most difference between this device and a conventional capacitor is the material of construction: the ultracapacitor dielectric is an electrolyte. If a voltage between the terminals is applied, a double layer appears between the electrodes and the electrolyte. As the distance between the charges is around armstrong, the capacity value per $\mathrm{cm}^{2}\left(\mu \mathrm{F} / \mathrm{cm}^{2}\right)$ is much higher than the corresponding one to a conventional capacitor $\left(\mathrm{nF} / \mathrm{cm}^{2}\right)$. Due to its high energetic density, the ultracapacitor can store efficiently the electric energy liberated during the regenerative braking, once the battery has been charged. Figure 5 shows a lineal dependence between the power supplied by the ultracapacitor and the charge state. The values of the power supplied are even $250 \mathrm{KW}$. Therefore, by means of this device, it is possible to cover the power range inaccessible by the fuel cell and the battery.

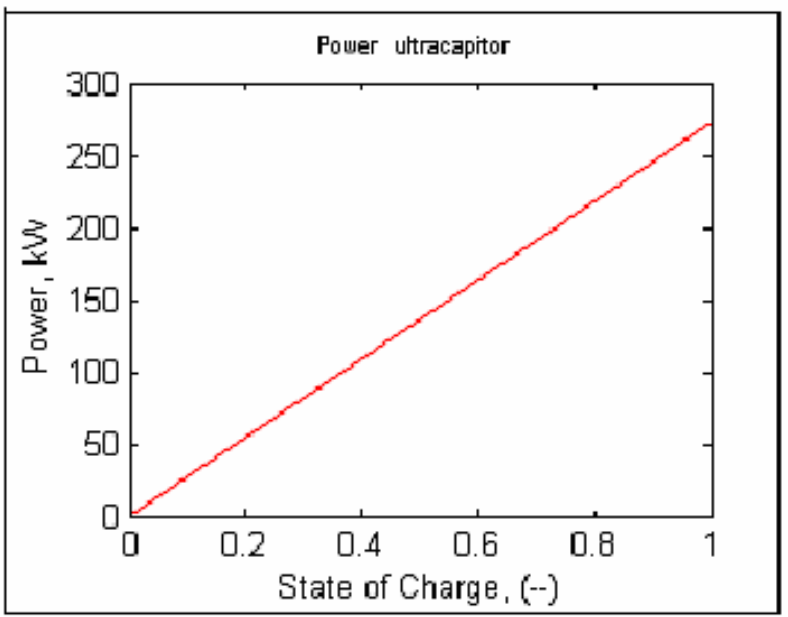

figure 5. Maximum Ultracapacitor Power 
The battery and the ultracapacitor recharge process is possible in phases in which the necessary power to make to work the vehicle is lower than the maximum one that the fuel cell can offer.

Another option is to charge the battery and the ultracapacitor coinciding with the moment of the braking, when the electrical engine works as generator (regenerative braking). The concept of regenerative braking arises when it is observed that it is possible to recover part of the energy that the electrical engine liberates when the variation of the speed regarding the one that circulates the vehicle starts being negative. The key is in controlling the time during that the inverter transistors are on, in order to establish a way so that the current flows from the electrical engine towards the store (battery and ultracapacitor). The battery recharge is carried out by a parallel circuit. The power flow from the electric motor to the battery can be controlled with a switch. The ultracapacitor recharge is carried out by the bidirectional DC/DC converter.

\section{Simulations}

National Renewable Energy Laboratory (NREL) has developed a vehicles simulator called ADVISOR (Advanced Vehicle Simulator), which is available since 1998 [4]. In this simulator, we can find an electric hybrid vehicle model with a fuel cell. The additional energy sources are only a battery. The simulation tests were carried out with a $1380 \mathrm{~kg}$ vehicle and a driving cycle named CYC_CLEVELAND, that is similar to a road cycle.

Figure 6 shows that there are differences between the demanded and the reached velocity, whose maximum values are around $30 \mathrm{~km} / \mathrm{h}$. This fact is caused by the energetic insufficiency presented in the vehicle configuration.

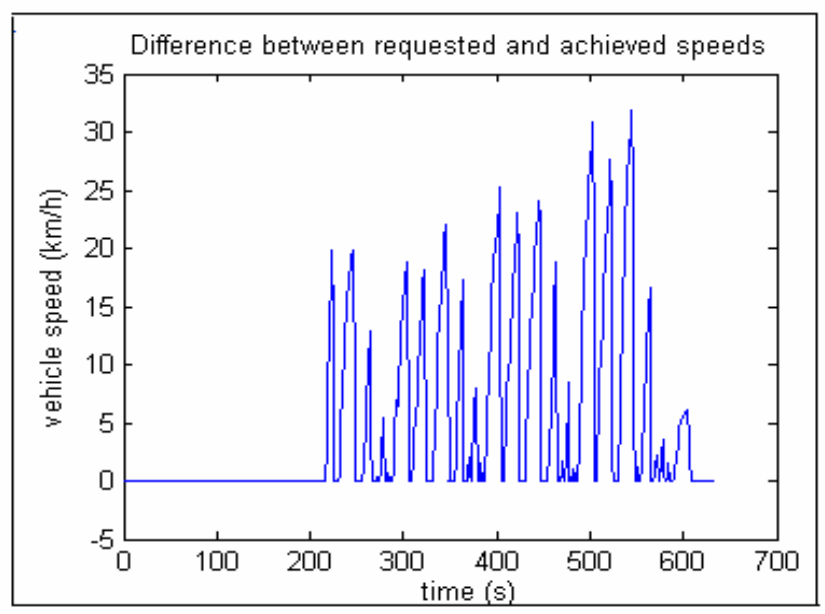

figure 6. Difference between requested and achieved speeds
The University of South California is also developing a simulation tool, Virtual Test Bed (VTB) [2], that allows to create virtual prototypes with different power sources and to connect different blocks easily.

A hybrid model with a fuel cell, a battery and a bank of ultracapacitors is available in this tool. Figure 7 shows the tests carried out with this model. The same parameters than the previous simulation were used. The results indicate that the difference between the demanded and the reached velocity is much smaller.

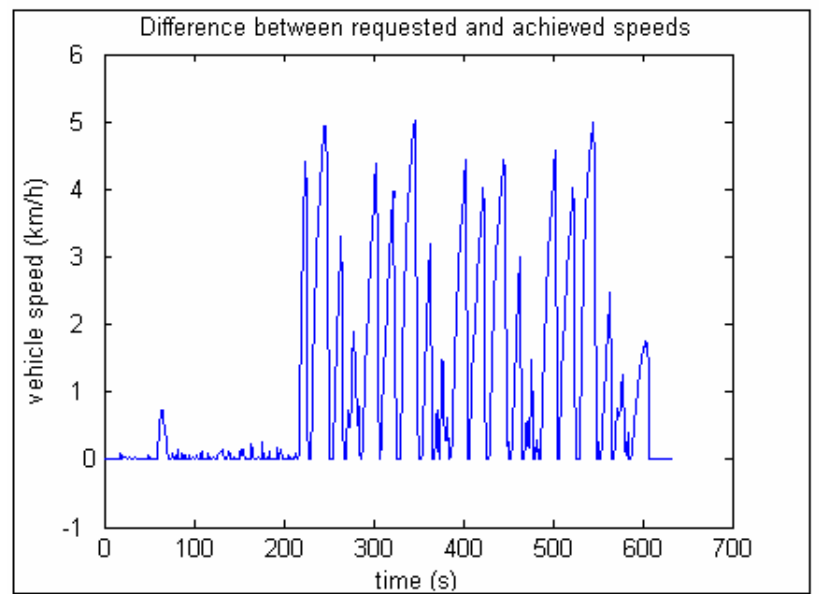

figure7. Difference between requested and achieved speeds

In the two cases, the control strategy consists on comparing the power required in the DC bus with the power available in each source. When the power required is bigger than the power available in the fuel cell, the battery supplies the difference.

¿Why not to use only a fuel cell with a bank of ultracapacitors? It was said that the bank of ultracapacitors offers high power levels in short time intervals. This does not guarantee the power provision during long intervals of average power demand, that are the most frequent in a real case of conduction. The election of the controller used to guarantee the distribution of energy at any moment and for any conduction profile, affects the hydrogen consumption and the optimal operation of the components. The algorithm that is often used is based on comparing the demanded power and the one that can provide each source, taking into account the fuel cell operation state and the battery charge state. Figure 8 shows how the fuel cell does not follow its curve of maximum efficiency. This result was obtained by means of ADVISOR using the series hybrid configuration with fuel cell and battery, without ultracapacitors, and the same parameters than the other simulations. 


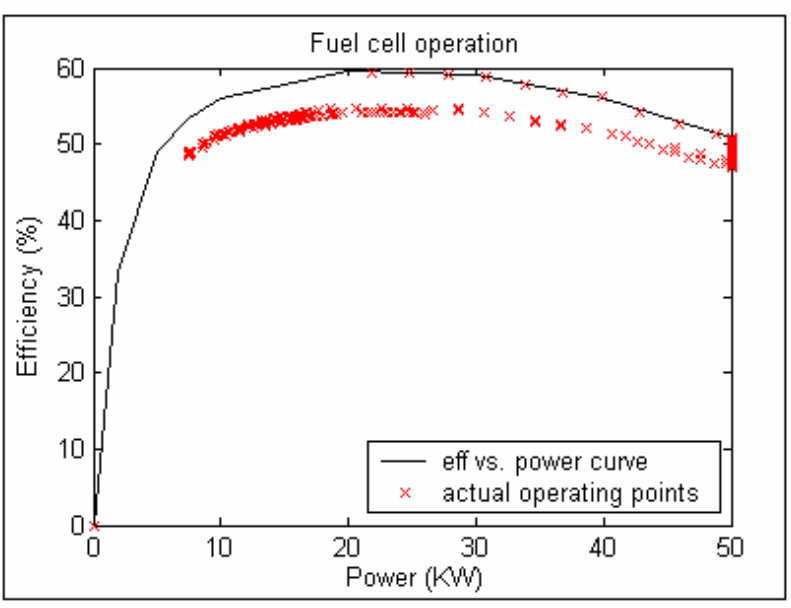

figure 8. Fuel Cell operation

On the other hand, it has been seen that the power provision is guaranteed with the three power sources. The next feature of the proposed topology that has to be verified is the energetic efficiency. Figure 9, obtained with VTB and the same parameters than previous simulations, shows how the operation points do not follow the maximum efficiency curve. Therefore, a new control strategy would be advisable

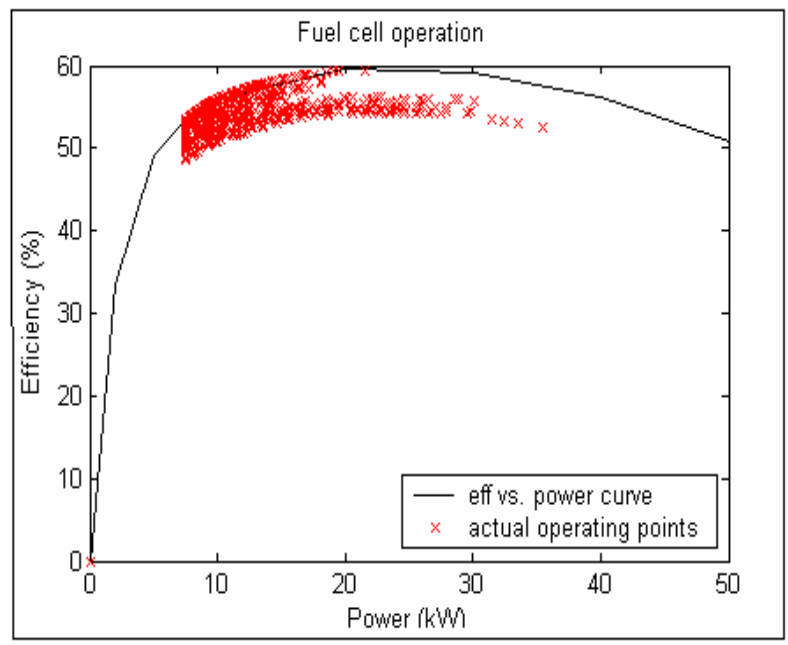

figure 9. Fuel Cell operation

\section{General scheme}

Figure 10 shows the connection scheme of all components.

\section{Energy Sources.}

The fuel cell and the bank of ultracapacitors are connected to the DC bus by $\mathrm{DC} / \mathrm{DC}$ converters because the output voltage of these components is not regulated.
The battery needs a converter because its output voltage is less that the specified bus voltage.

The power supply problem is solved controlling the fuel cell and the bank of the ultracapacitor converter. It means that, known the power value that the electric motor needs and the maximum value that each source can supply, the current reference is calculated for the fuel cell and for the ultracapacitor. Known this reference, the duty cicle of each converter would be calculated.

The battery is a device that supplies the whole current that is demanded if it is not higher than the limit fixed by the maker's parameters. The converter of the battery fixes the voltage in the DC bus. The difference between the real voltage and the reference voltage is obtained in order to calculate the duty cycle of the converter.

The DC bus ensures a constant input voltage in the inverter, which supplies the suitable voltage to the electric motor.

Control System.

The control strategy of the complete system, electric hybrid vehicle, will be carried out by means of a of fuzzy inference system. The design of the fuzzy control will be based on the models of each component of the vehicle, which are highly non-lineal. The models will be obtained by means of neurofuzzy techniques based on input/output data. The inputs to the controller will be the state of the fuel cell (temperature, pressure of the reagents in the anode and cathode, flow of hydrogen, etc.), the state of the auxiliary energy elements (state of battery charge, temperature of operation of the battery, state of charge of the ultracapacitor, temperature of operation of the ultracapacitor, etc.), the voltage and the current in the DC bus, the speed of the motor, the power demanded by the motor or the accelerator pedal command signal.

\section{Electric Motor.}

A comparative study reveals the advantages and disadvantages of the three classes of motors that can be used in the electric vehicles.

The induction motor (IM) has low efficiency, low power density, low noise, high overload capacity, great size, low cost and half maximum speed. The inverter to which the motor connects, presents low efficiency, complexity in the control, low power density and high cost.

The switch reluctance motor (SRM) has high efficiency, medium power density, high noise, medium/low overload capacity, medium size, medium cost and it is able to reach high speeds. The used converter has medium efficiency, medium power density, cost medium/low and it is not very complex regarding the control strategy.

The permanent magnetic motor (PM) is the one that presents bigger efficiency, bigger power density, reduced size and a medium maximum speed, that is in the range of revolutions allowed for automotive systems. The used 
converter has a high efficiency, medium control complexity, high power density and medium/low cost.

For transport aplications, a motor with reduced size/efficient rate and with high power density is looked for, therefore the permanent magnetic motor is chosen for this type of applications.

\section{Programmable load}

The programmable load consists on a electric generator that is connected to the electric motor. In it, the model of the vehicle is programmed. This model is equal to a group of equations that describe the effects related with the group of forces that is opposite to the movement of the vehicle (friction force, aerodynamics forces or gravitational force).

It would be interesting to include in the programmable load the power consumption corresponding with the vehicle electric charges (lights, radio set,...), as it does.

\section{Hydrogen storage.}

The storage of the hydrogen is carried out in tanks of steels of the type $\mathrm{Al}$ or $\mathrm{Ni}$, which are the less prone ones to the generation of breaks. There are also less heavy deposits made of composite and resins.

Chemists already know that the properties that make of the hydrogen a sure and useful fuel are the high energy density, the minimum activation energy, the high temperature of spontaneous combustion, the null emission of $\mathrm{CO} 2$, the high detonation limits, the low explosion energy and the high combustion speed. Moreover, it is not toxic.

The vehicle also needs to refuel in stations of electric recharge and to go to hydrogeners. In general, it is easier to establish a net of stations of hydrogen recharge taking advantage of the infrastructure of natural gas.

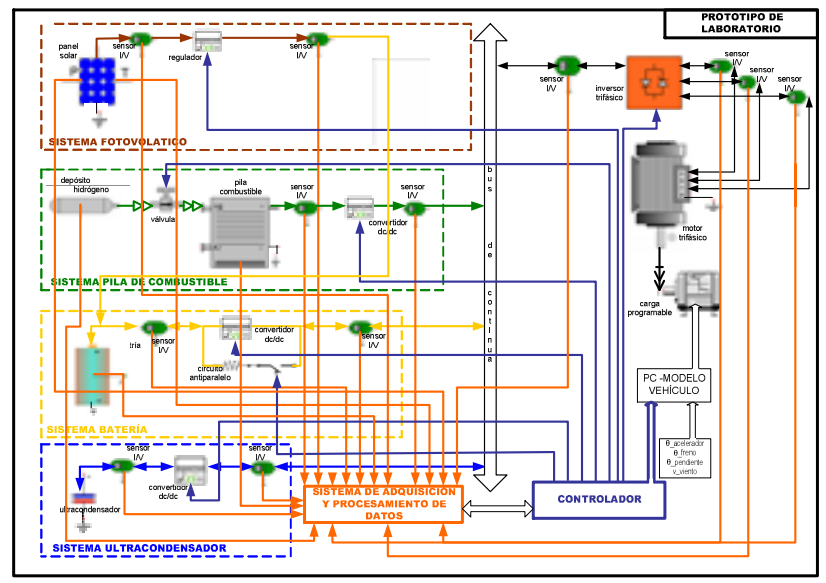

figure 10. General scheme

\section{Conclusions}

Several simulations show that the hybrid vehicle composed of a fuel cell and a battery is not enough to guarantee the energy supply. Nevertheless, the configuration composed of the three energy sources (the fuel cell, battery and bank of ultracapacitors) reduces the difference between the demanded and the supplied power.

It is also showed that fuel cell does not work in the maximum energy efficiency range, and so a new control strategy is justified.

Due to this reason, this new control strategy is being developed by the authors. Because of the very non-lineal behaviour of the components of the electric system, as well as the complexity of a suitable control so as to the system works efficienly, fuzzy control policies are being developed. These policies are based on machine learning of models by neuro-fuzzy techniques and on no-lineal control strategies [1].

\section{REFERENCES}

[1] Andújar, J. M., J. M. Bravo and A. Peregrín., "Stability analysis and synthesis of multivariable fuzzy systems using interval arithmetic", Fuzzy Sets and Systems,2004.

http://dx.doi.org/10.1016/j.fss.2004.01.008.

[2] Dougal R. A., Liu S., Gas L., Blackwelder M., "Virtual Test Bed for Advanced Power Sources",.Journal of Power and Sources,Vol.110, pp.285-295, 2002.

[3] Kheir, N. A., Schouten N. J., Salman M. A., "Fuzzy Logic Control for Parallel Hybrid Vehicles", IEEE Transaction on Control Systems Technology, Vol.10, No.3, pp.460-468, 2002.

[4] Markel T., Brooker A., Hendricks T., Jhonson V., Kelly K., Kamer B., O’Keefe M., Sprik S., Wipke K., "Advisor: a Systems Analysis Tool for Advanced Vehicle Modeling", Journal of Power and Sorces, Vol.110, pp.255-266. 2002. 